\title{
Embedded Fluorescence Lifetime Determination for High Throughput Real-Time Droplet Sorting with Microfluidics
}

\author{
Tobias Lieske*, Wilfried Uhring ${ }^{\dagger}$, Norbert Dumas ${ }^{\dagger}$, Jérémie Leonard ${ }^{\ddagger}$ and Dietmar Fey* \\ * Department of Computer Science, Chair of Computer Architecture \\ Friedrich-Alexander-University Erlangen-Nürnberg (FAU), Germany \\ $\{$ Tobias.Lieske, Dietmar.Fey\}@fau.de \\ ${ }^{\dagger}$ ICube, University of Strasbourg and CNRS, France \\ \{Wilfried.Uhring, Norbert.Dumas\}@icube.unistra.fr \\ †IPCMS, University of Strasbourg and CNRS, France \\ Jeremie.Leonard@ipcms.unistra.fr
}

\begin{abstract}
Time-resolved fluorescence (TRF) analysis is considered to be among the primary research tools in biochemistry and biophysics. One application of this method is the investigation of biomolecular interactions with promising applications for biosensing. For the latter context, time-correlated single photon counting (TCSPC) is the most sensitive, hence preferred implementation of TRF. However, high throughput applications are presently limited by the maximum achievable photon acquisition rate, and even more by the data processing rate. The latter rate is actually limited by the computational complexity to estimate accurately the fluorescence lifetime from TCSPC data. Here we propose a solution that would enable the implementation of TRF detection for fluorescence-activated droplet sorting (FADS), a particularly high throughput, microfluidic-based technology.

Most fluorescence lifetime algorithms require a large number of detected photons for an accurate lifetime computation. This paper presents an implementation based on a maximum likelihood estimator (MLE), enabling high precision estimation with a limited number of detected photons, significantly reducing the total measurement time. This speedup rapidly increases the input data rate. As a result, off-the-shelf embedded products cannot handle the data rates produced by current TCSPC units that are used to measure the fluorescence. Therefore, a configurable realtime capable hardware architecture is implemented on a fieldprogrammable gate array (FPGA) that can handle the data rates of future TCSPC units, rendering high throughput droplet sorting with microfluidics possible.
\end{abstract}

Keywords: FPGA, time-resolved fluorescence, fluorescence lifetime, microfluidics, embedded signal processing

\section{INTRODUCTION}

Fluorescence describes the emission of light from an electronically excited state. The lifetime $\tau$ is the average time between its excitation and the emission of a photon. Fluorescence spectroscopy and time-resolved fluorescence analysis methods are among the primary research tools in biochemistry and biophysics, but are not limited to these fields. The main biotechnological applications include flow cytometry, medical diagnostics, DNA sequencing, forensics and genetic analysis [1]. A typical example that benefits from increased throughput is fluorescence-activated droplet sorting (FADS) [2] that is used for drug discovery [3].
Fluorescence spectroscopy measures the average emission intensity, but results underlie many influences such as turbidity, sample concentration, geometry of the sample and chromophore bleaching. Furthermore, this analysis method cannot capture important information, such as fluorescence emitted by one fluorophore masking other fluorophores due to spectral overlap as well as interactions of the fluorophore with the substrate or other macromolecules [1].

Time-resolved fluorescence analysis methods on the other hand offer more information and are more robust, but require sophisticated optics and electronics due to the short time scale of fluorescence processes, which typically have emission rates of $10^{8} s^{-1}$ [1]. The analysis is based on the fluorescence lifetime, which has no dependency on emission intensity [4], [5] and is independent of the probe concentration as long as the probe emission is well above the background signal [1]. The fluorescence lifetime enables a distinct classification.

Microfluidics is a technology that allows the processing of small amounts of fluids $\left(10^{-9}\right.$ to $10^{-18}$ liters), utilizing channels with diameters of tens to hundreds of micrometers. This analysis technique offers various benefits such as carrying out separations and detections with high resolution and sensitivity, low cost and short times for analysis [6]. In order to combine the robustness of time-resolved fluorescence analysis with high throughput microfluidics, a sufficiently fast data processing is required to form a reliable high throughput sensing method. A particularly challenging example is microdroplet or cell sorting. The proposed experiment setup is illustrated in Fig. 1. A low cost high repetition rate picosecond laser diode generator is focused with lens $\mathrm{L}_{1}$ on the microdroplets in the channel, causing fluorescence. The emitted photons (dotted lines) pass the dichroic mirror unimpeded, are collected by lens $\mathrm{L}_{2}$ and then hit the single photon avalanche diode (SPAD). A time to digital converter (TDC) measures the time between the excitation and the emission of the photon. Based on this information, a histogram of arrival times can be generated, see Fig. 2. The fluorescence intensity is defined by Eq. (1), where $I_{0}$ is the initial intensity and $\tau$ the fluorescence lifetime [1].

$$
I(t)=I_{0} e^{-\frac{t}{\tau}}
$$




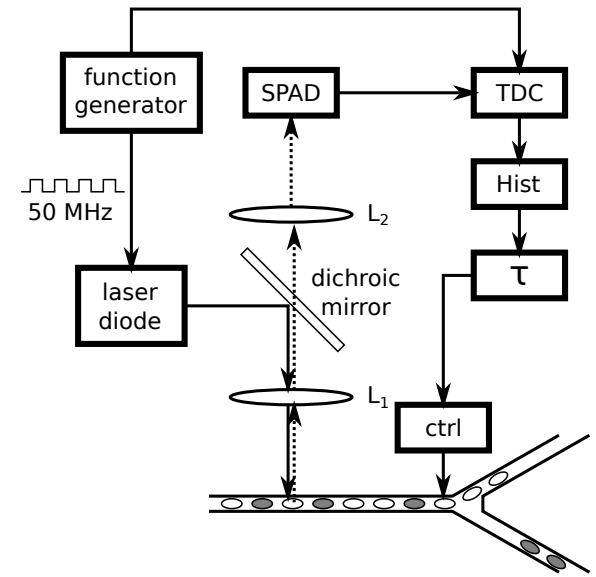

Fig. 1. Schematic setup of a microfluidics sorting system based on timeresolved fluorescence.

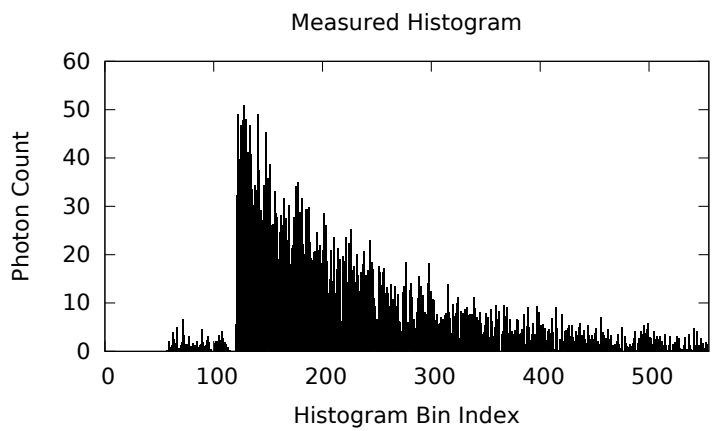

Fig. 2. Histogram of arrival times. Each bin represents a time slot and contains the number of photons detected in that slot.

The histogram of arrival times in Fig. 2 reflects the exponential decay character of the fluorescence intensity and can be used to extract the fluorescence lifetime $\tau$. The measured lifetime is then used to separate the classified microdroplets correctly further back in the channel. Due to the flow velocity, the fluorescence lifetime computation has a hard real-time deadline set at $5 \mathrm{~ms}$.

The challenge of this approach is the complexity of the time-resolved fluorescence analysis method. Microfluidics systems have no problem generating a laminar high throughput flow of up to twenty thousand so called microdroplets per second [7]. However, there are currently two problems that limit the actual throughput of such a sorting system. The photon counting rate for current time-correlated single-photon counting (TCSPC) systems is limited by the sample's lifetime, the pileup effect and dead-times of electronics, which will all be discussed in Section III. Established methods for fluorescence lifetime computation require large photon counts for precise estimation, resulting in a low throughput. Algorithms based on a maximum likelihood estimator (MLE) deliver reliable high precision results even with low photon counts per histogram. This drastically reduces the required measurement time and in turn increases the possible input data rate. Transferring the data to a powerful workstation consumes too much time and embedded off-the-shelf products cannot handle the resulting data rate. Therefore, a dedicated low latency, high throughput embedded hardware architecture for highly accurate fluorescence lifetime classification based on low signal-to-noise inputs has been developed. This new architecture renders high throughput microfluidic sorting possible with current TCSPC systems.

The following section reviews systems and methods for fluorescence lifetime extraction. After that, factors limiting the counting rate of TCSPC electronics for fluorescence measurements are explained in Section III and the MLE algorithm is revised in Section IV. Subsequently, the hardware architecture is explained in Section V and compared to existing approaches in Section VI. The paper ends with a brief conclusion in Section VII.

\section{RELATED WORK}

The following review focuses on the fluorescence lifetime extraction from a given histogram of arrival times, as displayed in Fig. 2.

The work presented in [8] shows a complete system similar to Fig. 1. While the TDC and histogram generation is implemented on a FPGA, the fluorescence lifetime is being computed externally on a workstation. Therefore, the histogram data is transferred via UART (Universal Asynchronous Receiver Transmitter). The results mention a delay of a few seconds to measure the fluorescence decay of a substance, which is clearly too slow for the real-time control system required by the sorting experiment proposed in this paper. Another approach using external processing is introduced in [9]. The fluorescence signals are transmitted via TCP/IP (Transmission Control Protocol/Internet Protocol) to a workstation that performs the lifetime computation. The signal acquisition, preprocessing and TCP/IP transmittance time is given as 9.5 ms. This time does not include the actual computation of the lifetime, proving that external computation of the fluorescence lifetime does not meet the given time constraints and an embedded approach is necessary to avoid long data transfer times.

There are several algorithms available that calculate the fluorescence lifetime. These algorithms can be classified into two groups. The first type are model based fitting methods such as Bayesian [10], maximum likelihood [11] or maximum entropy [12], method of moments [13] and least squares deconvolution with Laguerre expansion [9], [14]. These fitting methods return precise results, but have a relatively high computational complexity and easily converge to a local minimum. Furthermore, each model is derived from application knowledge such as the number of lifetimes contained in the data and thus the fitting approach is limited to the selected data [15].

The second algorithm class are nonfitting methods. Examples are the rotational invariance technique introduced in [15], the phasor analysis [16], Prony's method [17], the integral equation method [18] and the center of mass method [19], [20]. These algorithms offer a greatly reduced computational complexity when compared to the fitting based methods. Yet, some approaches are limited to monoexponential decays. An evaluation of these approaches is given in [15]. As will be shown in Section VI-B, the estimation quality of nonfitting methods is subpar and model based fitting methods offer superior results.

However, the main goal is to speed up the sorting process while maintaining the measurement precision. [11] shows that the maximum likelihood estimation (MLE) performs better on low signal-to-noise fluorescence decays than the popular least squares method and a classification of monoexponential decays 
is possible with very few photons [21]. In addition to that, the computational complexity for fluorescence lifetime estimation using the MLE algorithm is significantly lower than for the least squares algorithm [13]. Therefore, the MLE algorithm has been selected for further analysis, since collecting less photons per droplet results in a shorter measurement time and in a higher experiment throughput.

\section{COUnTING RATES OF TCSPC ELECTRONICS}

As already mentioned, the photon counting rate of TCSPC systems is limited by three factors. For one, TCSPC electronics exhibits a dead-time after a photon has been detected. Thus, photons arriving later at the SPAD can be masked by photons arriving early. This effect is called pulse pileup and deforms the recorded histogram in such a way that the measured fluorescence lifetime is reduced. This problem is usually addressed by following the $1 \%$ rule that states the excitation energy of one laser pulse should be so low that only one electron is emitted out of 100 excitation pulses. However, this $1 \%$ rule is being considered as overcautious. Counting rates at $10 \%$ decrease the lifetime by less than $1 \%$ and even a $30 \%$ count rate results only in a modest change [1]. Therefore, the technically maximum pulse repetition rate of the used laser can limit the photon counting rates. On the other hand, the maximum pulse repetition rate in order to determine the lifetime properly depends on the fluorescence decay times of the sample. The time between the pulses should be at least four times the longest lifetime in the sample [1]. Another technical limit is the actual duration of the dead-time of the TCSPC electronics. Effective counting rates further drop by $50 \%$ [1]. Considering an example with a lifetime of $5 \mathrm{~ns}$, a maximum laser repetition rate of $50 \mathrm{MHz}$ is allowed. Targeting a 30\% count rate results in 15 Mcps (counts per second), which can be handled by currently available TCSPC products [22]. As will be shown in Section VI-B, a total photon count of 500 per histogram is enough for precise lifetime extraction with an average error below $0.5 \%$ using the MLE algorithm. Provided one achieves a sufficiently fast fitting rate, up to 30000 microdroplets could be sorted per second. Our objective is therefore to implement a fitting routine that may process up to several $10^{4}$ histograms per second.

\section{FLUORESCENCE LIFETIME ESTIMATION}

The maximum likelihood estimator for monoexponential fluorescence decay signals has been derived in [13] and is given in Eq. (2). With $x=T / \tau, T$ the time step of one

$$
1+\left(e^{x}-1\right)^{-1}-m\left(e^{m x}-1\right)^{-1}=\bar{\nu}
$$

histogram bin, $m$ the number of histogram bins, $\bar{\nu}$ as defined in Eq. (3) and $N_{i}$ the number of photons in histogram bin $i$.

$$
\bar{\nu}=\frac{\sum_{i}^{m} i N_{i}}{\sum_{i}^{m} N_{i}}
$$

The term $\bar{\nu}$ can be seen as the histogram bin index with the "average" number of photons. The left-hand side of Eq. (2) is strictly monotone decreasing and admits a unique solution as long as the source is decaying. Therefore, a solution can be easily obtained by applying the Newton-Raphson method, as displayed in Eqs. (4) to (6).

Depending on the measured time window length, the total number of detected photons and the fluorescence lifetime, there

$$
\begin{aligned}
L(x) & =1+\left(e^{x}-1\right)^{-1}-m\left(e^{m x}-1\right)^{-1}-\bar{\nu}=0 \\
x_{i+1} & =x_{i}-\frac{L\left(x_{i}\right)}{L^{\prime}\left(x_{i}\right)} \\
& =x_{i}-\frac{1+\left(e^{x}-1\right)^{-1}-m\left(e^{m x}-1\right)^{-1}-\bar{\nu}}{m^{2} e^{m x}\left(m e^{m x}-1\right)^{-2}-e^{x}\left(e^{x}-1\right)^{-2}}
\end{aligned}
$$

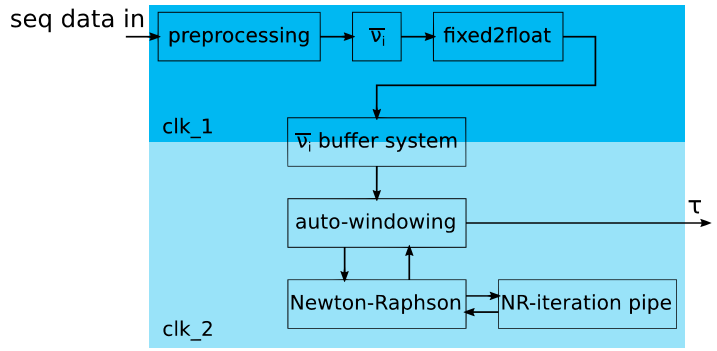

Fig. 3. System overview of the presented fluorescence lifetime computation unit.

is a visible noise floor, which can be seen in Fig. 2. As already mentioned, the photon count for the high throughput sorting application is relatively low. Therefore, higher index photon counts are relatively speaking stronger influenced by this noise floor, as these bins generally have a very low photon count. The noise floor deforms the exponential decay and directly influences $\bar{\nu}$ and thus the estimated fluorescence lifetime value $\tau$, see Eq. (3). Therefore, an iterative noise removal process called auto-windowing has been developed to acquire an improved approximation of the fluorescence lifetime. Depending on the estimated fluorescence lifetime, the input histogram is truncated to remove noisy bins and the fluorescence lifetime is recomputed, until a stable result is acquired.

This algorithm has been implemented in $\mathrm{C}$ on an $\mathrm{ARM}^{\circledR}$ Cortex ${ }^{\circledR}$-A53 quad-core processor. Processing one histogram of 1024 bins takes $152.365 \mu$ s. Assuming no delays caused by concurrent memory accesses, a total of 26.25 histograms per millisecond can be processed with this embedded processor, which is not sufficient to handle the data rate of 30 histograms per millisecond of the numerical example given in Section III. However, TCSPC electronics continuously improve enabling higher counting rates. Additionally, measuring a lifetime less than 5 ns allows higher laser repetition rates resulting in even higher counting rates, which in turn cannot be handled by the ARM $^{\circledR}$ Cortex $^{\circledR}-\mathrm{A} 53$ quad-core processor.

\section{HARDWARE IMPLEMENTATION}

Therefore, a dedicated hardware implementation has been developed in VHDL to avoid compute-bound fluorescence measurement systems and maximize the sorting throughput. The presented fluorescence lifetime computation system is displayed in Fig. 3 and structured in two parts. The first part addresses the input data and performs some preprocessing to compute $\bar{\nu}$ for Eq. (6). The second stage implements the Newton-Raphson iteration of the estimator function Eq. (6) to compute the fluorescence lifetime $\tau$. This pipeline-like system structure enables concurrent processing of input data.

\section{A. Preprocessing}

The preprocessing stage, dark colored part of Fig. 3, performs some noise suppression and computes $\bar{\nu}$ for every 
histogram bin according to Eq. (3). This is necessary since $\bar{\nu}$ depends on the histogram size and thus changes with the truncation process. In order to avoid saving the raw histogram input data and computing the required $\overline{\nu_{i}}$ on demand, all $\overline{\nu_{i}}$ values are precomputed and therefore are immediately available when the next auto-windowing iteration starts. The $\overline{\nu_{i}}$ computation uses fixed point arithmetic to save resources and is designed in a streaming fashion. The input histogram of arrival times is streamed in bin by bin sequentially.

While the preprocessing can be implemented in a fully pipelined streaming fashion, both the auto-windowing approach and the Newton-Raphson method are iterative algorithms. In order to maximize the throughput, the computed $\overline{\nu_{i}}$ values are stored in a buffer system as floating point values. The $\overline{\nu_{i}}$ values of different input histograms are stored in separate buffers and accessible to the second stage to enable concurrent computations of Eq. (6).

\section{B. Fluorescence Lifetime Computation}

The computation of the fluorescence lifetime is performed in the second stage of the system, see the light colored part of Fig. 3. Due to the large dynamic range of the exponential terms in Eq. (6), floating point values are used instead of fixed point. In order to avoid that the critical path of floating point arithmetic degrades the performance of the preprocessing stage, separate clocks are used for each stage and clock synchronization is implemented in the $\overline{\nu_{i}}$ buffer system module.

The second stage is fully pipelined, although autowindowing and Newton-Raphson are both iterative algorithms. The NR-iteration pipe block of Fig. 3 contains a fully pipelined implementation of Eq. (6) that can can be shared to compute several Newton-Raphson iterations in parallel. The autowindowing and Newton-Raphson blocks control the algorithm iterations and spawn new jobs for the NR-iteration pipe module. This way, up to 130 fluorescence lifetime computations can be performed in parallel each identified by a tag to ensure unambiguous assignment of input histogram to output lifetime. During computation, the necessary $\overline{\nu_{i}}$ values of the corresponding input histograms are stored in the $\overline{\nu_{i}}$ buffer system. The size of the $\overline{\nu_{i}}$ buffer system can be scaled statically to handle the input data stream without wasting resources. Further, the iteration limit for both the auto-windowing and the NewtonRaphson method can be configured statically (maximum) and dynamically to trade off precision and performance.

Using this system structure, multiple preprocessing stages can be connected to share one fluorescence lifetime computation stage to save resources.

\section{Evaluation}

\section{A. Implementation Results}

In order to evaluate the presented fluorescence lifetime computation system, a parameter set for the configuration has to be selected. Table I shows an excerpt of the static configuration parameters and their values used for the implementation results in Table II obtained by Quartus Prime 15.1.0. The Data Width defines the input bit width of a histogram bin represented as fixed point number. $m_{\max }$ configures buffer sizes and sets the maximum supported histogram size. AW Iter $r_{\max }$ configures the counter bit width for the auto-windowing iterations. The
TABLE I. CONFIGURATION PARAMETERS FOR IMPLEMENTATION RESULTS IN TABLE II.

\begin{tabular}{|c|c|c|c|c|}
\hline Data Width & $m_{\max }$ & AW Iter & NR Iter $_{\max }$ & \#Buffer \\
\hline 32 & 1024 & 10 & 10 & 16 \\
\hline
\end{tabular}

TABLE II. IMPLEMENTATION RESUlts ON ALTERA CyClONE V 5CSEMA4U23C6.

\begin{tabular}{|c|c|c|c|c|c|}
\hline$f_{\text {stage_1 }}$ & $f_{\text {stage_2 }}$ & FF & ALM & DSP & BRAM \\
\hline $155.18 \mathrm{MHz}$ & $147.45 \mathrm{MHz}$ & $29891(47 \%)$ & $11937(75 \%)$ & $71(85 \%)$ & $228(84 \%)$ \\
\hline
\end{tabular}

actual iteration count can be set dynamically between 1 and $A W$ Iter $_{\max }$. The same applies for the Newton-Raphson iteration

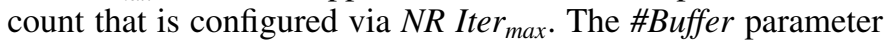
sets up the $\overline{\nu_{i}}$ buffer system.

An FPGA-based TDC and histogram generation module is introduced in [8]. Such a module can be implemented along with the here presented fluorescence lifetime computation system on the same FPGA chip, given the unused resources in Table II. Input histograms are streamed to the preprocessing stage bin by bin sequentially. Thus, the reported maximum clock frequency in Table II for the preprocessing stage corresponds to a potential input data stream of 151 histograms per millisecond, each histogram 1024 bins wide. Such a histogram rate may occur when the fluorescence lifetime needs to be measured at several points along the microfluidics channel or when counting rates of TCSPC electronics further improve.

Filling 1 of the 16 buffers in the $\overline{\nu_{i}}$ buffer system takes about $6.59 \mu$ s (1024 cycles). Assuming a continuous input data stream, the time window to process the data just stored in this buffer is about $98.98 \mu$ s (15360 cycles), which is the time to fill 15 buffers. Running the computation with the highest iteration count of 10 auto-windowing iterations, each consisting of 10 Newton-Raphson iterations, takes $93.86 \mu$ s (13840 cycles).

This means that even though an iterative algorithm has been chosen for the fluorescence lifetime computation, the presented architecture is IO bound due to smart parallel processing strategies. The given configuration can easily process 151 histograms per millisecond with a maximum computation delay of about $100,46 \mu$ s per histogram on a low cost FPGA development board [23]. The throughput and BRAM resource utilization of the system can be scaled down, by reducing the size of the $\overline{\nu_{i}}$ buffer system. In case higher input data rates need to be processed, several preprocessing stages can be connected with some glue logic to one shared computation stage.

\section{B. Quality of Computed Fluorescence Lifetimes}

In order to test the quality of the computed fluorescence lifetimes, input histograms for four fluorescence lifetimes $\tau \in\{1 \mathrm{~ns}, 3 \mathrm{~ns}, 5 \mathrm{~ns}, 15 \mathrm{~ns}\}$ have been generated. The time step $T$ and number of histograms bins $m$ have been selected according to Table III. Poisson noise has been added to the histograms, since this kind of noise is inherent for photon counting processes and also is the prominent kind of noise for low photon counts [1]. Further, the behavior for low photon counts needs to be analyzed. Therefore, histograms with a different total photon count $N \in\{500,600, \ldots, 1500\}$ have been generated. Due to the Poisson noise, the total number of photons deviates and is not exact for each histogram. For every $\{\tau, N\}$ set, 1000 histograms have been generated and the corresponding lifetime has been computed with the MLE algorithm implemented in $\mathrm{C}$ and by simulating the VHDL design. 


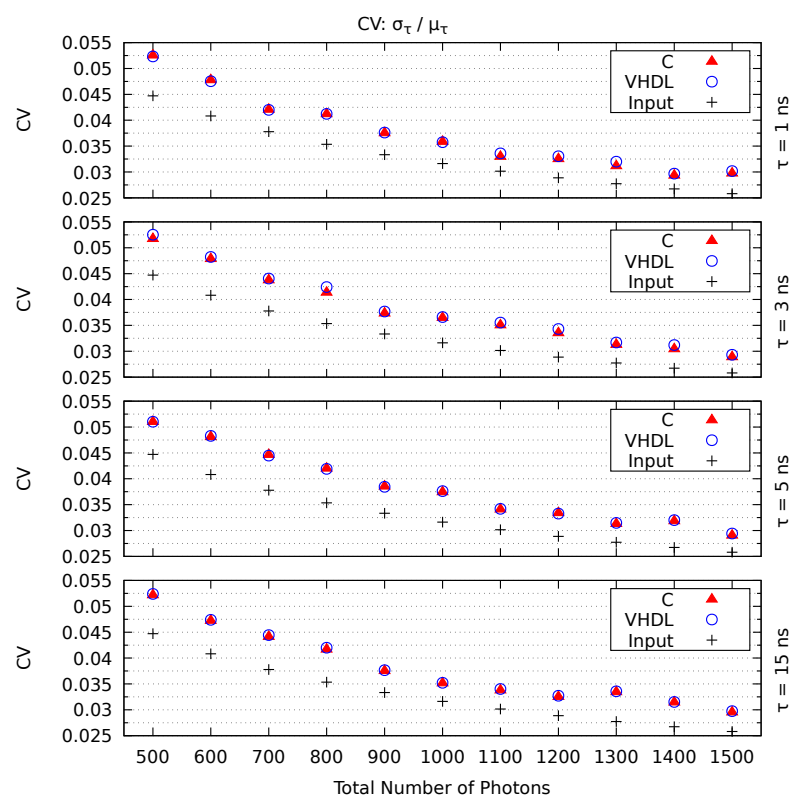

Fig. 4. Coefficient of variance for the $\mathrm{C}$ and VHDL implementation, as well for the input histogram.

TABLE III. TIME STEP AND HISTOGRAM SIZE FOR EACH $\tau$ TEST CASE.

\begin{tabular}{|c||r|r|r|r|}
\hline$\tau[\mathrm{ns}]$ & 1 & 3 & 5 & 15 \\
\hline$T[\mathrm{ps}]$ & 45 & 90 & 90 & 90 \\
\hline$m$ & 512 & 512 & 512 & 1024 \\
\hline
\end{tabular}

Figure 4 lists the coefficient of variance $(\mathrm{CV})$, which is the quotient of the standard deviation to the mean. This ratio is a standardized measure of dispersion. The results show that the noise introduced by the processing is in an acceptable range compared to the input noise ( $\mathrm{CV}$ of Poisson distribution is $1 / \sqrt{N}$ ) and decreases with increasing $N$. Slight variations are caused by the added Poisson noise. Some histograms are noisier than others and some sets include more of such "noisier" histograms. Similarly, $N$ varies from histogram to histogram.

The non fitting algorithms analyzed in [15] are compared by their normalized bias $(\Delta \tau / \tau)$ and the $F$-value, which is the normalized precision defined as $F=\sqrt{N} \cdot \sigma_{\tau} / \mu_{\tau}$. An ideal computation has $F=1$, while realistic computations feature $F>1$ and $F \gg 1$ [15]. The histograms used in [15] have $m=1024$ and a time bin width $T=12.5 \mathrm{~ns} / 1024$ $\approx 12.2 \mathrm{ps}$, which is a much finer temporal resolution than the one used for the test cases in this paper, see Table III. Unfortunately, $N$ is not given, but the number of photons in the first bin is, which is either 1000 when $\tau$ is being varied or $\tau$ is fixed at $3 \mathrm{~ns}$ and the number of photons in the first bin varies from 100 to 5000. In order to put these numbers into context, the mean photon count in the first bin where the exponential decay starts is listed in Table IV for all test cases used in this paper. It is obvious that the test cases used in this

TABLE IV. MEAN PHOTON COUNT IN THE FIRST BIN OF THE EXPONENTIAL DECAY.

\begin{tabular}{|r||r|r|r|r|r|r|r|r|r|r|r|}
\hline$\tau[\mathrm{ns}]$ & 500 & 600 & 700 & 800 & 900 & 1000 & 1100 & 1200 & 1300 & 1400 & 1500 \\
\hline \hline 1 & 22.38 & 27.00 & 31.61 & 34.88 & 40.09 & 43.34 & 48.86 & 52.15 & 57.67 & 61.78 & 67.17 \\
\hline 3 & 13.34 & 16.56 & 19.19 & 21.85 & 24.50 & 27.10 & 29.24 & 33.07 & 35.16 & 37.88 & 41.23 \\
\hline 5 & 8.83 & 10.35 & 11.89 & 13.80 & 15.63 & 16.89 & 18.84 & 20.77 & 22.18 & 24.05 & 25.63 \\
\hline 15 & 2.97 & 3.46 & 4.10 & 4.60 & 5.24 & 5.89 & 6.57 & 7.31 & 7.68 & 8.24 & 8.86 \\
\hline
\end{tabular}

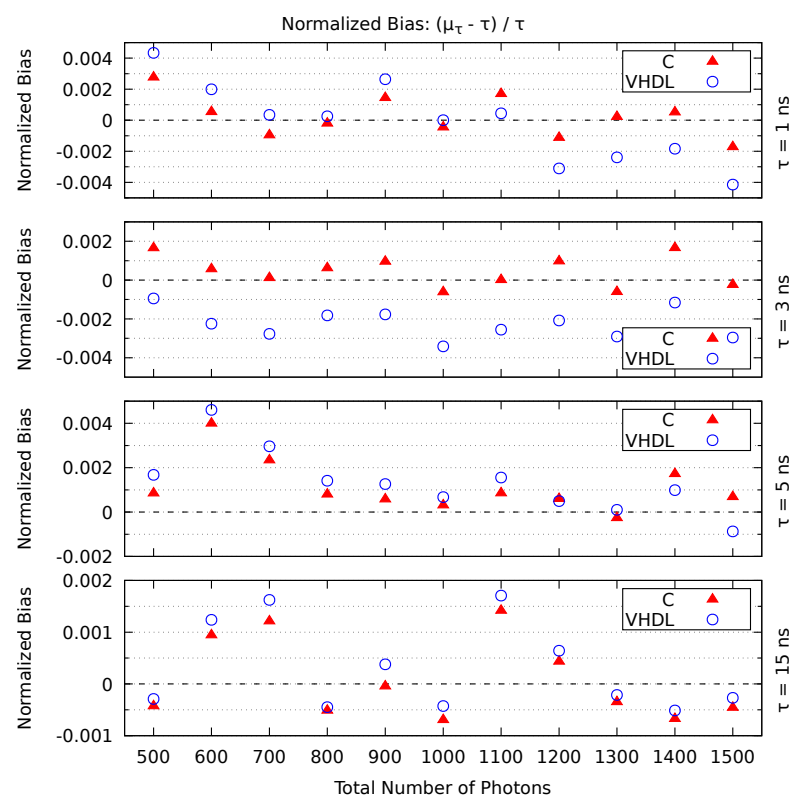

Fig. 5. Normalized bias for $\mathrm{C}$ and VHDL implementation. The optimum is a bias of 0 .
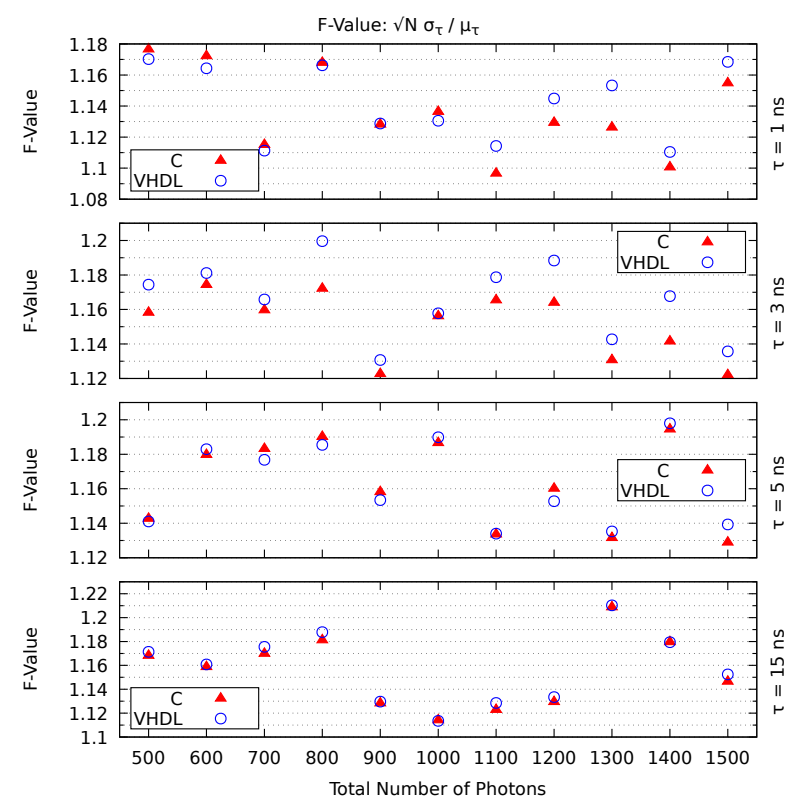

Fig. 6. F-Value for $\mathrm{C}$ and VHDL implementation.

paper feature a drastically lower total photon count $N$, which implies a much shorter measurement time per droplet and thus improves the throughput. Nonetheless, the resulting bias and F-value measurements presented in Figs. 5 and 6 respectively, show that the MLE algorithm returns superior results.

The bias for all measurements plotted in Fig. 5 is clearly below $0.5 \%$, while the bias reported in [15] for $\tau=3 \mathrm{~ns}$ for the integral equation method (IEM) is about $-1.6 \%$ and about $6.3 \%$ for the center of mass method (CMM). Both clearly exceeding the bias of the MLE. Further, the listed phasor analysis features a bias of about $0.8 \%(\tau=3 \mathrm{~ns})$, while the presented fluorescence lifetime estimation via rotational invariance techniques (FLERIT) algorithm is very close to $0 \%$ bias $(\tau=3 \mathrm{~ns})$. Extracting an exact range from the plot is not possible due to 
the coarse scale. The CMM method is discarded for the further comparison considering its excessive lifetime dependent bias.

Looking at the F-values reported in [15], it is clear that FLERIT, IEM and phasor exhibit a lifetime dependent precision. The authors list optimized regions for the analyzed algorithms, where $F<4$. These regions are from 0.4 ns to $14 \mathrm{~ns}$ for FLERIT, $0.4 \mathrm{~ns}$ to $8.8 \mathrm{~ns}$ for IEM and $0.4 \mathrm{~ns}$ to $4.23 \mathrm{~ns}$ for phasor. A higher resolution plot for $\tau=3 \mathrm{~ns}$ shows that the F-value for the two best algorithms FLERIT and IEM varies between 1.5 and 2.0 [15]. In contrast to this, the F-value measurements of the MLE algorithm given in Fig. 6 show no dependency on the lifetime and no test case exceeds a F-value of 1.22 .

\section{CONCLUSION}

Time-resolved fluorescence analysis is among the primary research tools in biochemistry and biophysics [1]. Increasing the throughput of FADS systems improves the efficiency of drug discovery and can help finding new cures for diseases. This paper presents the implementation of a MLE based fluorescence measurement system. The results show that the algorithm offers superior fluorescence lifetime estimations, even for very low signal-to-noise inputs, i.e. low photon count. This enables shorter measurement times, which in turn increases the throughput of FADS systems, while also improving the measurement quality.

The MLE algorithm features indeed a higher computational complexity than non fitting algorithms. However, this paper shows that a flexible low cost FPGA implementation is capable of processing high data rates in real time.

\section{ACKNOWLEDGEMENT}

This work was financially supported by the Research Training Group 1773 “Heterogeneous Image Systems”, funded by the German Research Foundation (DFG).

\section{REFERENCES}

[1] J. R. Lakowicz, Principles of Fluorescence Spectroscopy, 3rd ed. Springer US, 2006.

[2] J.-C. Baret, O. J. Miller, V. Taly, M. Ryckelynck, A. El-Harrak, L. Frenz, C. Rick, M. L. Samuels, J. B. Hutchison, J. J. Agresti, D. R. Link, D. A. Weitz, and A. D. Griffiths, "Fluorescence-activated droplet sorting (fads): efficient microfluidic cell sorting based on enzymatic activity," Lab Chip, vol. 9, pp. 1850-1858, 2009. [Online]. Available: http://dx.doi.org/10.1039/B902504A

[3] L. Schmid, D. A. Weitz, and T. Franke, "Sorting drops and cells with acoustics: acoustic microfluidic fluorescence-activated cell sorter," Lab Chip, vol. 14, pp. 3710-3718, 2014. [Online]. Available: http://dx.doi.org/10.1039/C4LC00588K

[4] C. R. Giles, C. A. Burrus, D. J. DiGiovanni, N. K. Dutta, and G. Raybon, "Characterization of erbium-doped fibers and application to modeling 980-nm and 1480-nm pumped amplifiers," IEEE Photonics Technology Letters, vol. 3, no. 4, pp. 363-365, April 1991.

[5] R. Paschotta, J. Nilsson, A. C. Tropper, and D. C. Hanna, "Ytterbiumdoped fiber amplifiers," IEEE Journal of Quantum Electronics, vol. 33, no. 7, pp. 1049-1056, Jul 1997.

[6] G. M. Whitesides, "The origins and the future of microfluidics," Nature, vol. 442, no. 7101, pp. 368-373, Jul 2006. [Online]. Available: http://dx.doi.org/10.1038/nature05058

[7] S.-Y. Teh, R. Lin, L.-H. Hung, and A. P. Lee, "Droplet microfluidics," Lab Chip, vol. 8, pp. 198-220, 2008. [Online]. Available: http: //dx.doi.org/10.1039/B715524G
[8] N. Franch, O. Alonso, J. Canals, A. Vil, A. Herms, and A. Dieguez, "A low cost fluorescence lifetime measurement system based on spad detectors and fpga processing," in 2016 Conference on Design of Circuits and Integrated Systems (DCIS), Nov 2016, pp. 1-6.

[9] D. Gorpas, D. Ma, J. Bec, D. R. Yankelevich, and L. Marcu, "Realtime visualization of tissue surface biochemical features derived from fluorescence lifetime measurements," IEEE Transactions on Medical Imaging, vol. 35, no. 8, pp. 1802-1811, Aug 2016.

[10] M. I. Rowley, P. R. Barber, A. C. C. Coolen, and B. Vojnovic, "Bayesian analysis of fluorescence lifetime imaging data," pp. 790325-79032512, 2011. [Online]. Available: http://dx.doi.org/10.1117/12.873890

[11] M. Maus, M. Cotlet, J. Hofkens, T. Gensch, F. C. De Schryver, J. Schaffer, and C. A. M. Seidel, "An experimental comparison of the maximum likelihood estimation and nonlinear least-squares fluorescence lifetime analysis of single molecules," Analytical Chemistry, vol. 73, no. 9, pp. 2078-2086, 2001, pMID: 11354494. [Online]. Available: http://dx.doi.org/10.1021/ac000877g

[12] R. Swaminathan and N. Periasamy, "Analysis of fluorescence decay by the maximum entropy method: Influence of noise and analysis parameters on the width of the distribution of lifetimes," Proceedings of the Indian Academy of Sciences - Chemical Sciences, vol. 108, no. 1, p. 39, 1996. [Online]. Available: http://dx.doi.org/10.1007/BF02872511

[13] P. Hall and B. Selinger, "Better estimates of exponential decay parameters," The Journal of Physical Chemistry, vol. 85, no. 20, pp. 2941-2946, 1981. [Online]. Available: http://dx.doi.org/10.1021/ j150620a019

[14] J. Liu, Y. Sun, J. Qi, and L. Marcu, "A novel method for fast and robust estimation of fluorescence decay dynamics using constrained least-squares deconvolution with laguerre expansion," Physics in Medicine and Biology, vol. 57, no. 4, p. 843, 2012. [Online]. Available: http://stacks.iop.org/0031-9155/57/i=4/a=843

[15] H. Yu, R. Saleeb, P. Dalgarno, and D. D.-U. Li, "Estimation of fluorescence lifetimes via rotational invariance techniques," IEEE Transactions on Biomedical Engineering, vol. 63, no. 6, pp. 1292-1300, June 2016.

[16] M. A. Digman, V. R. Caiolfa, M. Zamai, and E. Gratton, "The phasor approach to fluorescence lifetime imaging analysis," Biophys $J$, vol. 94, no. 2, pp. L14-L16, Jan 2008, 120154[PII]. [Online]. Available: http://www.ncbi.nlm.nih.gov/pmc/articles/PMC2157251/

[17] Z. Zhang, K. T. V. Grattan, Y. Hu, A. W. Palmer, and B. T. Meggitt, "Pronys method for exponential lifetime estimations in fluorescencebased thermometers," Review of Scientific Instruments, vol. 67, no. 7, pp. 2590-2594, 1996. [Online]. Available: http: //dx.doi.org/10.1063/1.1147219

[18] D.-U. Li, J. Arlt, J. Richardson, R. Walker, A. Buts, D. Stoppa, E. Charbon, and R. Henderson, "Real-time fluorescence lifetime imaging system with a $32 \times 320.13 \mu \mathrm{m}$ cmos low darkcount single-photon avalanche diode array," Opt. Express, vol. 18, no. 10, pp. 10257-10269, May 2010. [Online]. Available: http: //www.opticsexpress.org/abstract.cfm?URI=oe-18-10-10257

[19] D. D.-U. Li, J. Arlt, D. Tyndall, R. Walker, J. Richardson, D. Stoppa, E. Charbon, and R. K. Henderson, "Video-rate fluorescence lifetime imaging camera with cmos single-photon avalanche diode arrays and high-speed imaging algorithm," Journal of Biomedical Optics, vol. 16, no. 9, pp. 096012-096012-12, 2011. [Online]. Available: http://dx.doi.org/10.1117/1.3625288

[20] D. Tyndall, B. R. Rae, D. D. U. Li, J. Arlt, A. Johnston, J. A. Richardson, and R. K. Henderson, "A high-throughput time-resolved minisilicon photomultiplier with embedded fluorescence lifetime estimation in $0.13 \mu \mathrm{m}$ cmos," IEEE Transactions on Biomedical Circuits and Systems, vol. 6, no. 6, pp. 562-570, Dec 2012.

[21] M. Köllner and J. Wolfrum, "How many photons are necessary for fluorescence-lifetime measurements?" Chemical Physics Letters, vol. 200, no. 1, pp. 199 - 204, 1992. [Online]. Available: http://www.sciencedirect.com/science/article/pii/000926149287068Z

[22] TimeHarp 260, Rudower Chaussee 29, 12489 Berlin, Germany, 2016. [Online]. Available: http://www.picoquant.com/images/uploads/ downloads/timeharp260.pdf

[23] DE0-Nano-SoC User Manual, 9F., No.176, Sec.2, Gongdao 5th Rd, East Dist, Hsinchu City, 30070. Taiwan, 2015. [Online]. Available: http://www.terasic.com.tw/attachment/archive/941/ DE0-Nano-SoC_User_manual.pdf 